Optimization of satellite orbit for SVLBI obs.

\title{
The optimization of satellite orbit for Space-VLBI observation
}

\author{
Lei Liu $^{1,2,3}$, Weimin Zheng ${ }^{1,2,3}$ \\ ${ }^{1}$ Shanghai Astronomical Observatory, Chinese Academy of Sciences; liulei@shao.ac.cn, \\ zhwm@shao.ac.cn \\ ${ }^{2}$ Key Laboratory of Radio Astronomy, Chinese Academy of Sciences, Nanjing 210008, China \\ ${ }^{3}$ Shanghai Key Laboratory of Space Navigation and Positioning Techniques, Shanghai 200030, China
}

\begin{abstract}
By sending one or more telescopes into space, Space-VLBI (SVLBI) is able to achieve even higher angular resolution and is therefore the trend of the VLBI technique. For SVLBI program, the design of satellite orbits plays an important role for the success of planned observation. In this paper, we present our orbit optimization scheme, so as to facilitate the design of satellite orbit for SVLBI observation. To achieve that, we characterize the $u v$ coverage with a measure index and minimize it by finding out the corresponding orbit configuration. In this way, the design of satellite orbit is converted to an optimization problem. We can prove that, with appropriate global minimization method, the best orbit configuration can be found within the reasonable time. Besides that, we demonstrate this scheme can be used for the scheduling of SVLBI observations.
\end{abstract}

Key words: instrumentation: interferometers — techniques: high angular resolution methods: numerical — space vehicles: instruments

\section{INTRODUCTION}

VLBI (Very Long Baseline Interferometry) is the astronomical instrument with the highest angular resolution, and is therefore widely used in the field of astrophysics (Event Horizon Telescope Collaboration 2019), astrometry (Ma et al. 2009; Schuh \& Behrend 2012) and deep space exploration (Duev et al. 2012; Zheng et al. 2014). The resolution of VLBI is proportional to the baseline length and the observation frequency (Thompson et al. 2001). For ground based VLBI, the length of a baseline is limited by the size of the Earth. E.g. the Event Horizon Telescope achieved an angular resolution of $23 \mu$ as at $230 \mathrm{GHz}$ with baseline length comparable to the Earth diameter (Event Horizon Telescope Collaboration 2019). To achieve even higher angular resolution at the given frequency, one natural choice is to send one or more telescopes into space, which is the so called Space-VLBI (SVLBI; Gurvits 2018).

Japan sent the first VLBI satellite VSOP (VLBI Space Observatory) into space in 1997 (Hirabayashi et al. 1998, 2000). It was equipped with an 8.8 meter parabola antenna and works in 1.6 and $5 \mathrm{GHz}$. The orbit height was $22,000 \mathrm{~km}$. The mission came to an end in 2005. Another SVLBI program was RadioAstron by Russia. It was launched in 2011 and worked until 2019. The designed observation frequencies were 0.3, 
1.6, 5, $22 \mathrm{GHz}$. The orbit height was $338,000 \mathrm{~km}$, which helped the project achieve the highest angular resolution of $7 \mu$ as at $22 \mathrm{GHz}$ for SVLBI observations (Kardashev et al. 2012).

Several SVLBI projects are under development in China (An et al. 2020). Shanghai Astronomical Observatory (SHAO) proposed the mission concept of space mm-wavelength VLBI array (SMVA) in 2010s (Hong et al. 2014). With the support of Chinese Academy of Sciences, prototype studies are conducted for the technical feasibility of the mission. One of the main achievement is the $10-\mathrm{m}$ space antenna prototype (Hong et al. 2014; Liu et al. 2016).

At present, SHAO is proposing a new SVLBI project: the Space Low Frequency Radio Observatory. In this project, two satellites each equipped with a 30 meter radio telescope will be sent into the Earth elliptical orbit (orbit height 2,000 km $\times 90,000 \mathrm{~km}$ ). The observation frequency ranges from $30 \mathrm{MHz}$ to 1.7GHz. Two telescopes will conduct collaborate observation with FAST (Five-hundred-meter Aperture Spherical radio Telescope), SKA (Square Kilometer Array) and other ground based $100 \mathrm{~m}$ level large radio telescopes, so as to achieve both high angular resolution and sensitivity. This project is special, as two satellites dedicated to SVLBI observations make it standout from VSOP and RadioAstron, of which only one satellite is deployed. Also it is different from Chang'E missions, for which the orbit is fixed and will not be adjusted for SVLBI observations. For the first time, the project will provide unprecedented flexibility for the design of satellite orbit dedicated to VLBI observations. For VLBI observation, one of the most important applications is to obtain the high angular resolution image of the target (radio imaging). In this process, a good $u v$ coverage is essential for obtaining an ideal antenna beam, and finally determines the quality of the image. However, this is not a trivial task. First of all, by looking through literature, one may realize that there are no commonly accepted rules for the characterization of a "good" $u v$ coverage that is suitable for radio imaging. Moreover, a satellite orbit is uniquely described by 6 orbital elements. Orbit design for two satellites involves the combination of 12 such kind of elements. It is actually computationally challenging to find out the orbit configuration that yields the best "uv" coverage from the large parameter space.

The design of satellite orbit for SVLBI observations is somewhat similar to the classical array configuration problem that has been well studied in the last three decades. Although the trajectory of a space telescope is quite different from that of ground based telescopes, we can still gain inspirations from previous work. Keto (1997) propose the array shape of a curve with constant width, so as to achieve a uniform sampling in the $u v$ plane. Boone (2001) argued that a Gaussian radial and uniform angular distribution in the $u v$ plane yielded a Gaussian shaped synthesis beam. Therefore, they optimized the array based on the discrepancy (the "pressure force") between the model and the actual coverage. Kogan (1997) took another approach by minimizing the side lobes. Su et al. (2004) optimize the array distribution by taking a "thieving" approach. Karastergiou et al. (2006) characterized the $u v$ coverage with a single quantity and go through all possible array configurations to minimize it.

In this paper, we try to solve the orbit design problem by drawing inspirations from previous work and taking into account the orbit configuration. In short, we characterize the $u v$ coverage with a measure index and minimize it by finding out the corresponding satellite orbit configuration. In this way, orbit design 


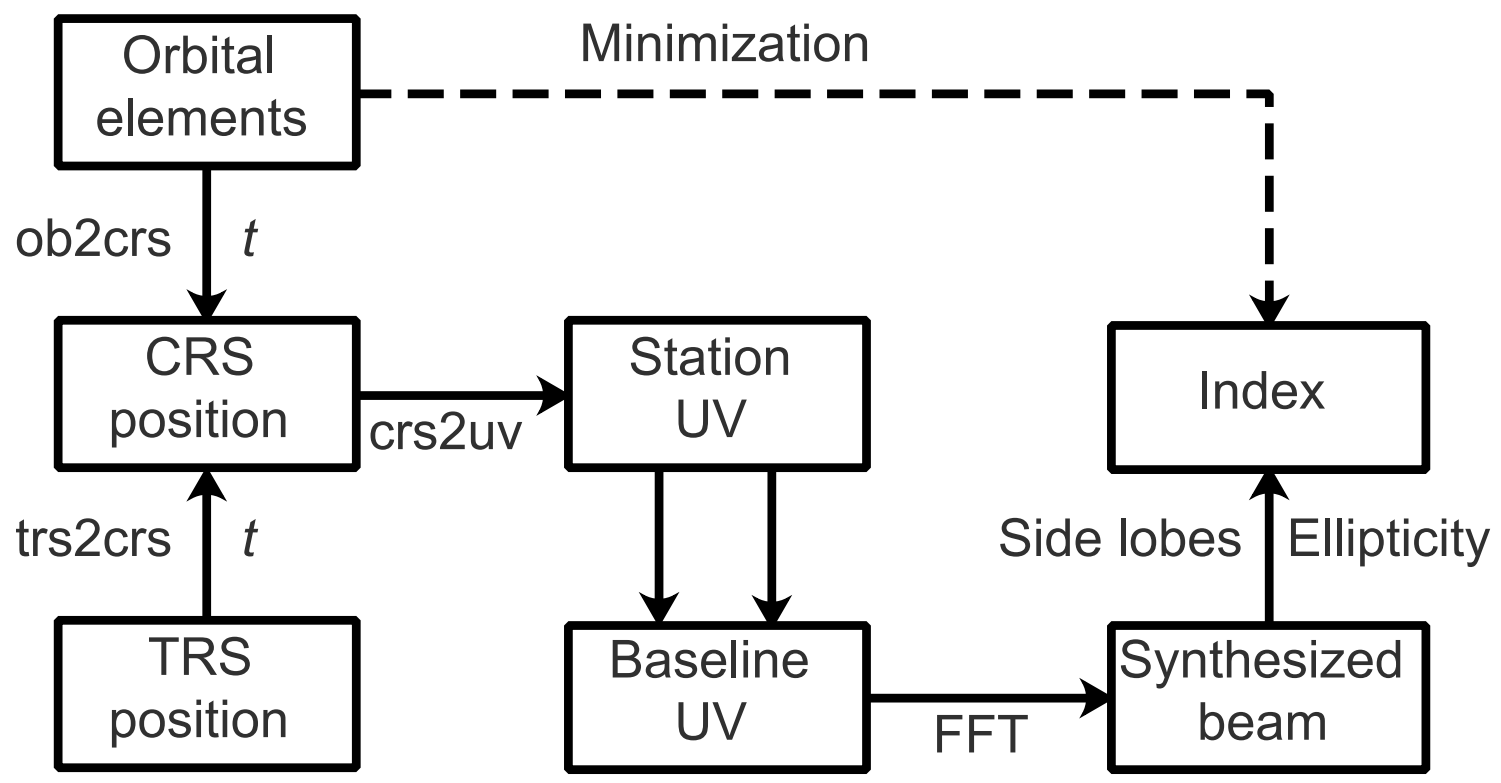

Fig. 1: Data flow chart of the orbit design and optimization scheme.

is converted to an optimization problem. Our work proves that this approach is feasible, the best orbit configuration can be found within a reasonable time using modern global minimization method.

This paper is organized as follows: in Sec.2 we introduce the optimization scheme; in Sec. 3 we present its application, including the design of orbit and the schedule of observation. In Sec.4 4 we discuss the results and present summary and conclusions.

\section{THE ORBIT DESIGN AND OPTIMIZATION SCHEME}

In this work, we characterize the $u v$ coverage with an index $s=s(u v)$. Given a specific time range, the $u v$ coverage is determined by the configuration of satellite orbit. 1 According to the classical satellite dynamics, the orbit of a given celestial object is described by 6 elements: semi-major axis $a$, eccentricity $e$, inclination $i$, right ascension of ascending node $\Omega$, argument of periapsis $\omega$ and mean anomaly at reference epoch $M_{0}$. Accordingly, for two satellites in the planned array there will be 12 such kind of elements. As a result, the index can be expressed as an optimization function of those elements. In this way, the design of satellite orbits is converted to an optimization problem.

The data flow of the optimization scheme is demonstrated in Fig. 11 For each given moment of time $t$, the telescope position (or state vector) is calculated in Celestial Reference System (CRS). For satellite, this routine is based on the orbital elements. For ground based stations, transformation of station coordinates from TRS (Terrestrial Reference System) to CRS is conducted. In this process, we take into account the Earth rotation, precession, nutation and polar motion effects 2. Once the station CRS positions are obtained, we calculate their projections on the $u v$ plane, and further calculate the baseline $u v$. The next step is to characterize the $u v$ coverage with an index, which is based on the synthesized beam in the image plane. For radio imaging, a good $u v$ coverage yields a smooth synthesized beam, which is crucial for the quality

1 It is actually the trajectories of satellites and ground stations together determine the $u v$ coverage. However, the latter part only depends on earth rotation and is fixed at given time range. The variation of $u v$ coverage is determined by orbit configurations.

2 The precession, nutation and polar motion matrices require earth orientation parameters, which are routinely updated by IERS. 

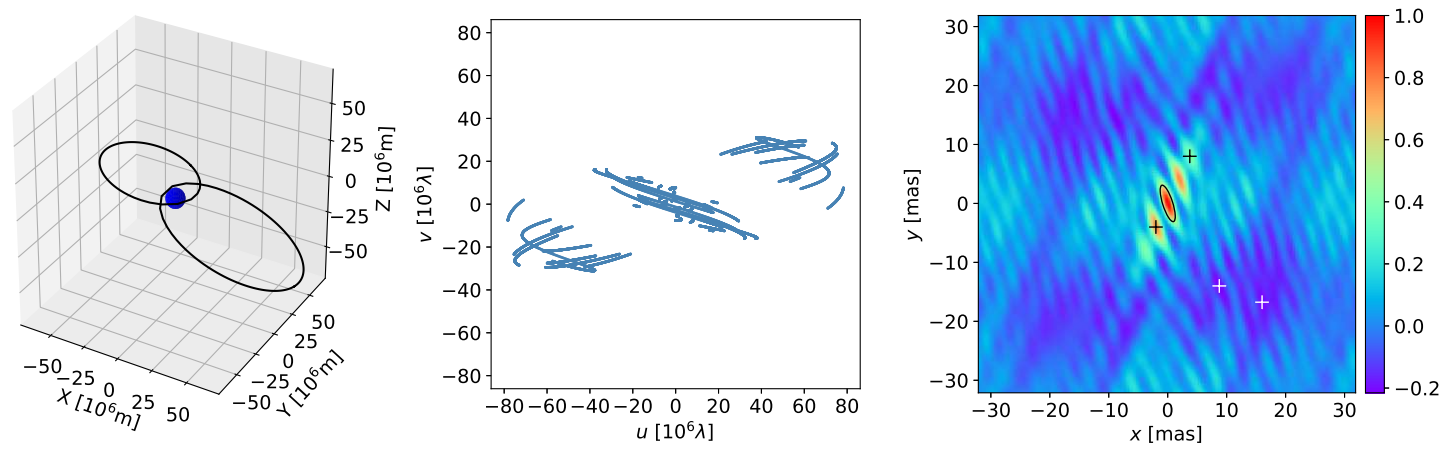

Fig. 2: Orbit configuration (left), $u v$ coverage (middle) and beam pattern (right) before optimization. The main lobe of the beam is shown with a black ellipse. Orbital elements are selected randomly by the optimization function. The first and second peaks and nadirs of the side lobe are marked with black and white crosses, respectively. Index: 1.718193 (L1), semi-major axis: 52,378.1 km, eccentricity: 0.84, inclination: $-21.4^{\circ} / 6.3^{\circ}$, right ascension of ascending node: $-134.3^{\circ} / 129.3^{\circ}$, argument of periapsis: $-51.2^{\circ} / 92.4^{\circ}$, mean anomaly: $163.5^{\circ} / 38.9^{\circ}$.
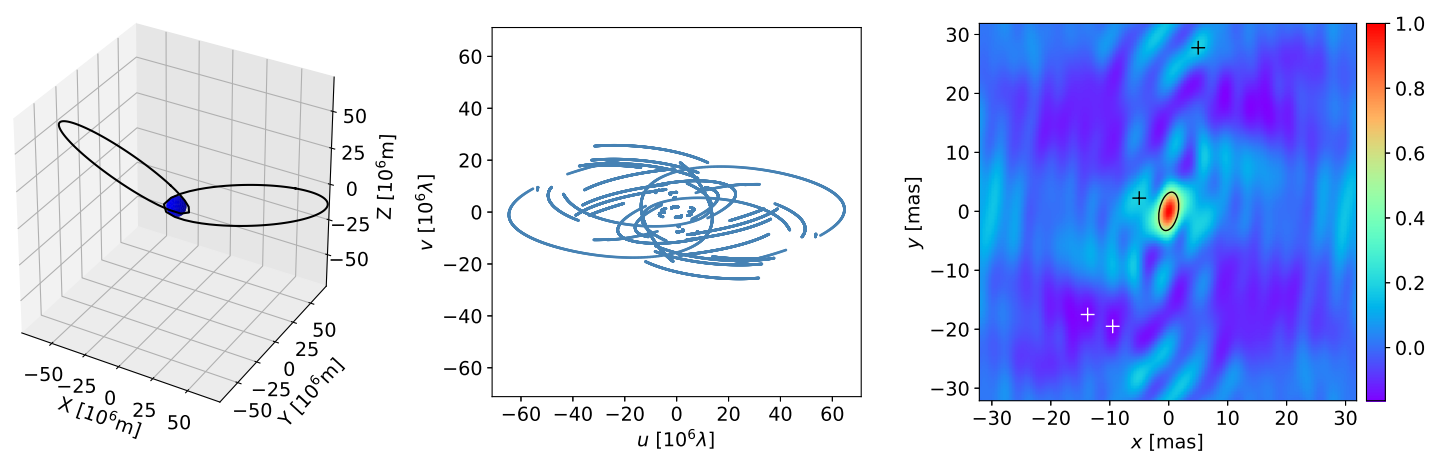

Fig. 3: Orbit configuration (left), $u v$ coverage (middle) and beam pattern (right) after optimization with L1 norm. Descriptions of ellipse and crosses are provided in the caption of Fig. 2. Index: 0.749449 (L1), semimajor axis: $52,378.1 \mathrm{~km}$, eccentricity: 0.84 , inclination: $151.5^{\circ} / 4.6^{\circ}$, right ascension of ascending node: $-41.3^{\circ} / 45.9^{\circ}$, argument of periapsis: $-38.8^{\circ} / 163.6^{\circ}$, mean anomaly: $-19.5^{\circ} /-91.0^{\circ}$.

of the final image. The details on the index calculation are provided in the next section. To this point, the index is actually a function of satellite orbital elements. Thus, it would be possible to find the best orbit configuration by minimizing the index value.

\subsection{Characterization of $u v$ coverage}

The key idea of this work is to find a quantity that characterizes the $u v$ coverage appropriately. In our view, it must fulfill the following requirements:

- Scalar form. Suitable for minimization.

- Accurate. Smaller value yields better coverage.

- Easy to calculate. Since the calculation will be conducted many times when investigating the huge parameter space, calculation speed is very important. 

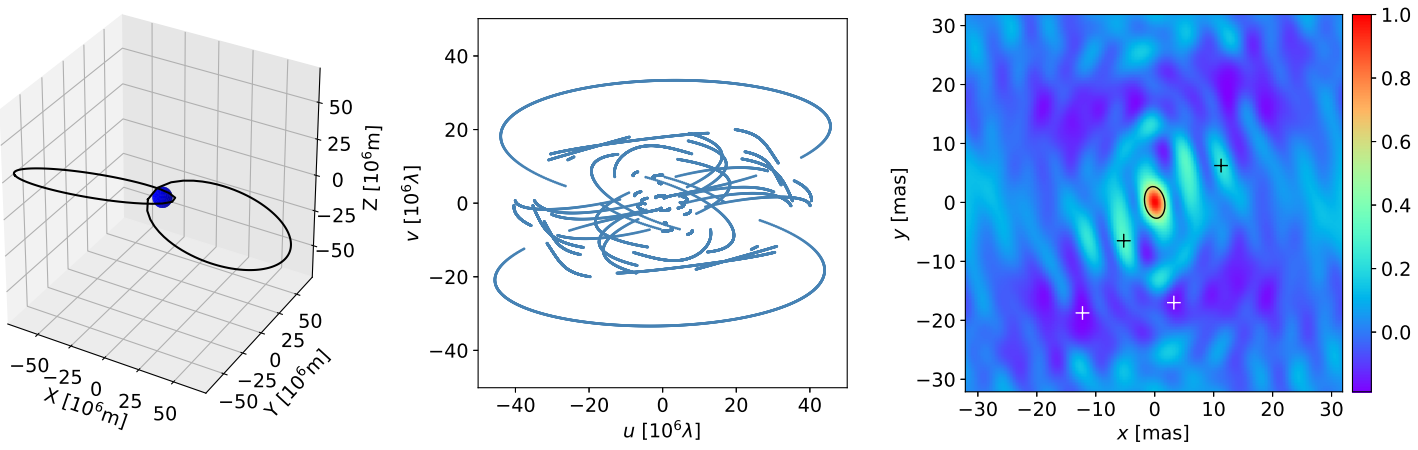

Fig. 4: Orbit configuration (left), $u v$ coverage (middle) and beam pattern (right) after optimization with L2 norm. Meanings of ellipse and crosses are explained in the caption of Fig. 2. Index: 0.488062 (L2), semi-major axis: $52,378.1 \mathrm{~km}$, eccentricity: 0.84 , inclination: $-163.1^{\circ} / 162.5^{\circ}$, right ascension of ascending node: $-177.7^{\circ} / 4.8^{\circ}$, argument of periapsis: $159.3^{\circ} / 167.1^{\circ}$, mean anomaly: $153.5^{\circ} / 2.1^{\circ}$.

We draw inspirations from previous work. Initially, we took the idea of Boone (2001) and used an index to evaluate the discrepancy between a Gaussian shaped radial distribution and actual data. Soon we realized that the Gaussian distribution was difficult to achieve for SVLBI observations of which the $u v$ coverage is usually sparse. Actually this is already pointed out by Karastergiou et al. (2006) that Boone (2001) scheme is mainly for dense interferometer. Eventually we decided to characterize the $u v$ coverage with the synthesized beam in the image plane. Although the $u v$ coverage and the synthesized beam are mathematically equivalent, the latter one is relatively easier to evaluate: an ideal beam should be smooth and round (less fluctuate and oblate) in shape. We propose a measure index of the following form:

$$
s_{\mathrm{L} 1}=w_{r} r_{\mathrm{L} 1}+w_{e} e .
$$

Here $r_{\mathrm{L} 1}=\left(a_{1}+a_{2}+\left|a_{-1}\right|+\left|a_{-2}\right|\right) / a_{0}$ is the ratio of side lobes to the main lobe, which measures the fluctuation of the beam. $a_{i}$ represent the value of the $i$-th peak/nadir of the beam pattern. $a_{0}$ is the main lobe. $a_{1}$ and $a_{2}$ are the first and the second side lobes. $a_{-1}$ and $a_{-2}$ are the first and the second nadirs, which are negative. $e=b_{\mathrm{maj}} / b_{\min }-1$ measures the ellipticity of the beam. $b_{\mathrm{maj}}$ and $b_{\min }$ are the major and minor axis of the beam, which are derived from the $u v$ coverage with TPJ's algorithm in DIFMAP (Shepherd 1997). $w_{r}$ and $w_{e}$ are the weights of the two terms. In this work, we set them to 0.9 and 0.1 , respectively. Initially the ellipticity of the beam is not taken into account. Soon we realize that this might lead to an extremely oblate beam (large ellipticity). According to our test, a weight of 0.1 for the ellipticity term effectively reduces the oblateness of the beam in the optimization process. We have to point out that our choice of weights is somewhat arbitrary. Their values could be further adjusted to achieve a better optimization result in the actual application. Our optimization approach is similar with Kogan (1997), which adjust antenna positions to reduce the side lobes. One may find that the sum of absolute values correspond to the L1 norm. It is also worth investigating the $\mathrm{L} 2$ norm:

$$
s_{\mathrm{L} 2}=w_{r} r_{\mathrm{L} 2}+w_{e} e .
$$

Here $r_{\mathrm{L} 2}=\left(a_{1}^{2}+a_{2}^{2}+a_{-1}^{2}+a_{-2}^{2}\right)^{1 / 2} / a_{0}$. 


\subsection{Constrains of Space-VLBI observation}

The space low frequency array project is still in its preliminary stage, which gives us a lot of flexibility to design the orbit. However, VLBI is a complex technique, placing one or more antennas in the Earth orbit introduces many extra uncertainties and makes orbit design even more difficult. As a result, there are still some constraints that must be taken into account. We list them below and discuss their influence on orbit design.

- Orbit height. According to the preliminary plan, the VLBI satellite will be sent into the Earth elliptical orbit by rocket. In principle, the rocket itself has no special requirement for the orbit. For VLBI consideration, the apogee height is set to $90,000 \mathrm{~km}$, such that the baseline length is one order magnitude longer than that of the ground based VLBI. Meanwhile, the perigee height is fixed at 2,000 km, so as to guarantee a data transmission rate of $1.5 \mathrm{Gbps}$ in $\mathrm{X}$ or Ka band. These constraints fix the semi-major axis and the eccentricity, which reduce the parameters from 12 to 8 and therefore speedup the optimization process.

- Observation time. Having the orbit height such as mentioned above, the corresponding orbit period will be 33.1 hours. To obtain a good $u v$ coverage, observations to the target source should cover the whole elliptical orbit. However, it is not realistic to require that the observation is continuous in the whole orbit period. As a result, observations should be conducted several times when the satellite is located at different parts of the orbit.

- Collaboration with ground based telescopes. When conducting VLBI observations, two satellites will collaborate with the ground based large telescopes, so as to achieve both high angular resolution and sensitivity simultaneously. When simulating $u v$ coverage, contributions from ground-ground, spaceground baselines must be taken into account and will determine the final beam patterns together with space-space baselines. As a result, their presence will affect on the selection of orbital elements in the optimization process.

\subsection{Implementation of optimization scheme}

Concerning the complex relationship between satellite orbit and the corresponding $u v$ coverage, the optimization function cannot be described analytically. Moreover, it is not guaranteed that the function is convex, which means there might be many local minima that must be avoided when looking for global minima. Optimization is a large topic in applied mathematics. It is completely not our intention to develop an optimization method from scratch for our work. Fortunately, there are many well developed global minimization methods which have been implemented in Python scipy package. Among them we choose the “differential_evolution" method (Storn \& Price 1997). According to our test, it is able to find out the solution (global minimum) within the reasonable time(around $15 \mathrm{~min}$ for each solution), as we will describe in detail in Sec.3.1.2. We have to point out that this does not necessarily mean it outperforms other global optimization methods in the algorithmic level. According to our analysis, the main reason it converges faster than other methods is it provides parallel implementation in current scipy package. 


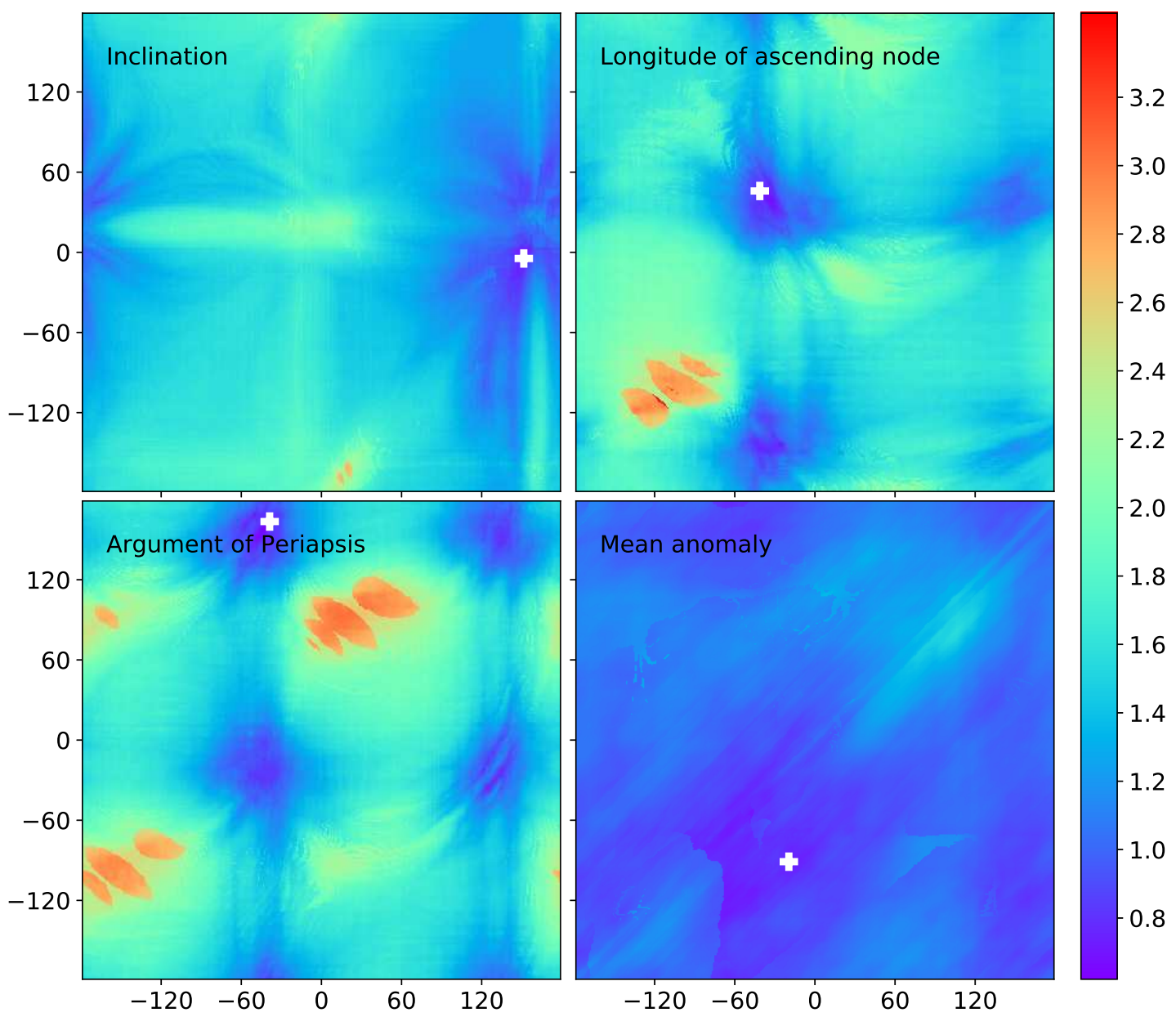

Fig. 5: Snapshots of the optimization function in the phase space. Index values at each pixel are calculated by investigating the 2 parameters labeled on the top left of the corresponding panel while keeping other 6 parameters set to the L1 solution (see Fig. 3 for the value). The pixel size of the 4 panels is set to $1^{\circ}$. White cross in each panel labels out the solution found by the differential_evolution method.

\section{APPLICATIONS}

In this section, we present two applications of the optimization scheme: orbit design and observation scheduling, as described below.

\subsection{Orbital design}

\subsubsection{Observation setup}

We setup an observation for the application of the orbit design scheme. The assumed observation starts on 2020-03-11T00:00:00 UTC and lasts for 24 hours. The target source is M87 (Park et al. 2019). The observation frequency is set to $300 \mathrm{MHz}$. According to the preliminary plan, two VLBI satellites take part in the observation. Five ground based telescopes, FAST (Five-hundred-meter Aperture Spherical radio Telescope; Nan et al. 2011), QTT (QiTai radio Telescope), Effelsberg $100 \mathrm{~m}$ adio telescope in Germany, GBT (Green Bank Telescope) in US and the planned SKA-low in Australia take part in the observation. 
FAST has conducted VLBI observations successfully with TMRT (TianMa Radio Telescope in Shanghai) last year. We are expecting more scientific breakthrough with its extremely high sensitivity. QTT is still in its construction stage, which is promising in conducting collaborated observation in the next 10 years. The minimal elevation angle is set to $30^{\circ}$ for FAST, and $15^{\circ}$ for other ground based telescopes. Besides that, to avoid radio interference from the Earth, we set a minimum separation angle of $5^{\circ}$ between the source and the Earth surface at the satellite.

\subsubsection{Optimization result}

We use the "differential_evolution" method provided by scipy package to find out the global minimum of the optimization function. The calculation of $u v$ coverage and the localization of zenith/nadir point of the beam pattern in each set of orbit configuration is a time consuming process. To keep a reasonable optimization speed, positions of satellites and ground based telescopes are sampled every 1 minute. For the beam pattern, the cell size is set to 0.25 mas, which is around $1 / 10$ of the angular resolution for a $100,000 \mathrm{~km}$ baseline at $300 \mathrm{MHz}$. The apogee and perigee heights are set to $90,000 \mathrm{~km}$ and 2,000 km, respectively. This fixes the semi-major axis and the eccentricity of the orbit. As a result, the optimization is conducted in the 8 dimensional parameter space composed of inclination, longitude of ascending node, argument of Periapsis and mean anomaly of the two satellites. Based on our actual implementation, it takes about 15 minutes on 12 workers (processes) in a server equipped with 4 Intel Xeon E7530 @ $1.87 \mathrm{GHz}$ (24 physical cores in total) for each optimization in the paper. We have to emphasize that this result is strongly depends on the hardware platform.

Fig. 2, 3 and 4 demonstrate the orbit, $u v$ coverage and beam pattern before and after optimization. The orbital elements (solutions) are presented in the caption of the corresponding figures. By observing the main lobe and the surrounding region, we may find that the optimized result is much more smooth and less oblate. This is consistent with our design of the optimization function. The orbit configuration selected by the optimization routine yields the smallest index. We may expect that compared with the unoptimized beam, the optimized Gaussian shaped beam is more suitable for deconvolution. Also note that the minor axis of the beam pattern corresponds to an elongated $u v$ distribution in the same direction, and vice verse. This is consistent with the radio imaging theory: the angular resolution is proportional to the inverse of the baseline length $(\theta \sim \lambda / d)$. We have to point that although the $u v$ coverage of the optimized orbit is much better than that of the unoptimized, the baseline length of the optimized orbit is shorter, which means we obtain a good beam pattern at the expense of lower angular resolution. However, we think this is worthwhile since a small beam with large fluctuation is of no help for imaging. We have to point out that such kind of long baselines might be necessary in some non-imaging applications, e.g., high angular resolution astrometry. If we care only about the position and the target is a point source, a little bit worse beam pattern is not a problem and can be overcome (Liu et al. 2019).

\subsubsection{The complex structure of phase space}

Fig. 5 present the snapshots of the optimization function in the phase space. The purpose of these snapshots is to demonstrate the complex structures of the parameter phase space. The 8 parameters are tangled together 


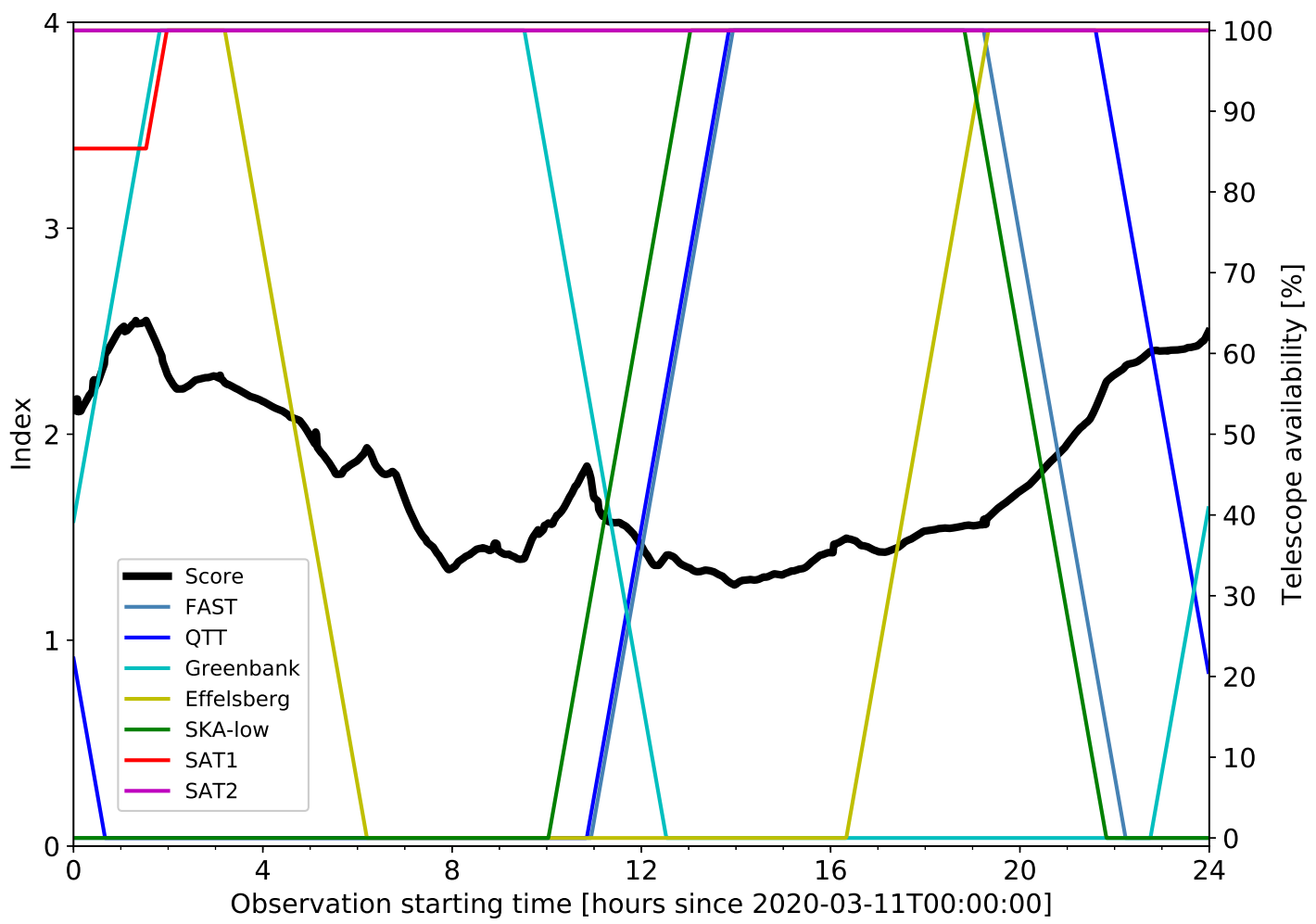

Fig. 6: The index as a function of observation starting time. The planned observation is a 3 hours session for M87. Thick black line and thin colored lines correspond to the index and telescope availabilities, respectively.

in a highly non-linear way, which makes the localization of the minimum point a difficult task: the global optimization method should avoid getting trapped in the "local minimum". Our work proves that with an appropriate method, the global minimum solution can be found within the reasonable time.

\subsection{Observation scheduling}

\subsubsection{Motivation}

Another possible application of the optimization scheme is observation scheduling. There are already mature VLBI schedule programs for ground based telescopes. E.g., "Sked" (Gipson 2010) and "VieSched++" (Schartner \& Böhm 2019) for geodetic observations, "Sched' 3 for astrophysical observations. However, the scheduling of SVLBI observations is still a blank area. For SVLBI, the position of VLBI satellite is determined by the orbit configuration instead of the Earth rotation. Besides that, the calculation of telescope availability is quite different from that of ground based telescopes. The eclipse of the Earth, data storage and many other space related ingredients must be taken into account. Moreover, the $u v$ coverage of SVLBI observations is usually poor. More attention should be paid to the resulting beam. All of these make the schedule of SVLBI observations quite different from that of ground based observations. As a result, mature schedule methods and programs cannot be used directly in SVLBI observations. It is very necessary to develop a new method that takes the features of SVLBI observations into account.

3 http://www.aoc.nrao.edu/software/sched/ 


\subsubsection{Scheduling result}

We have found that our characterization of the $u v$ coverage and optimization scheme is very suitable for the scheduling of SVLBI observation. E.g., given some time for a source, what is the best time range to conduct the observation? Our answer is the scheduling can be converted to an optimization problem. This is demonstrated in Fig. 6, for a 3 hours observation, the index that characterizes the $u v$ coverage is a function of observation starting time: the observation yields the small index if it starts at 14:00 when 2 satellites and 3 ground based telescopes are fully available for observation. This is consistent with a basic scheduling principle: to utilize as many telescopes in the observation as possible. All of above suggest that our characterization of the $u v$ coverage is very helpful in SVLBI scheduling.

\section{CONCLUSIONS AND DISCUSSIONS}

In this paper, we present our orbit optimization scheme for the design of VLBI satellite orbit. In this scheme, we characterize the $u v$ coverage with an index and minimize it by finding out the corresponding orbit configuration. In this way, the design of satellite orbit is converted to an optimization problem. To valid the scheme, we setup a 24 hours observation for M87. Five large ground based telescopes FAST, QTT, Greenbank, Effelsberg, SKA-low and two VLBI satellites take part in the observation. Although the structure of the optimization parameter phase space is complex, we can prove that with modern global minimization method, it is possible to find out the best orbit configuration within the reasonable time. Moreover, we demonstrate that our characterization of $u v$ coverage can be used for the scheduling of SVLBI observations.

We want to point out that the optimization scheme could and should be improved continuously. First of all, current optimization function (index) is based on our understanding of a good $u v$ coverage, which deals only with the fluctuation and the ellipticity of the beam pattern. However, in actual application more parameters should be taken into account, e.g., angular resolution, sensitivity, etc. All of these would contribute to the optimization function with appropriate weights. Besides that, in this work, we only demonstrate the optimization for one source. For an actual scientific project, the optimization function should be the combination of a list of target sources. As long as we have obtained adequate computational resources, it is not difficult for our scheme to take multiple sources into account. Moreover, for a real satellite, there will be definitely more constraints on the orbit configuration. E.g., orbit height, inclination, etc. Our scheme provides enough flexibility to include these constraints in the optimization process.

The design of satellite orbit for SVLBI observation is a blank area. We still have a long way to go to obtain a commonly accepted "good" orbit configuration. We hope our work is helpful for China's future SVLBI project.

Acknowledgements This work is supported by the National Natural Science Foundation of China (Grant Nos. 11903067, 11973011, 11573057, U1831137, 11703070), Shanghai Outstanding Academic Leaders Plan, the Strategic Priority Research Program of the Chinese Academy of Sciences, grant No. XDB23010200, Shanghai Key Laboratory of Space Navigation and Positioning Techniques (ZZXT201902). We would like to show our deep appreciation to Dr. Wu Jiang, Dr. Jongho Park, Ms. Yidan Huang, Mr. Xingfu Liu for their kind support in writing this paper. We thank the referee for suggestions that greatly helped to improve the quality of this paper. 


\section{References}


An, T., Hong, X., Zheng, W., et al. 2020, Advances in Space Research, 65, 850

Boone, F. 2001, A\&A, 377, 368

Duev, D. A., Molera Calvés, G., Pogrebenko, S. V., et al. 2012, A\&A, 541, A43

Event Horizon Telescope Collaboration, Akiyama, K., Alberdi, A., et al. 2019, ApJL, 875, L1

Gipson, J. 2010, Sixth International VLBI Service for Geodesy and Astronomy. Proceedings from the 2010 General Meeting, 77

GurvitsL. I., 2018, Proceedings of the 69th International Astronautical Congress (IAC-18-A 7.2.8), Bremen, 1 - 5 Oct 2018.

Hirabayashi, H., Hirosawa, H., Kobayashi, H., et al. 1998, Science, 281, 1825

Hirabayashi, H., Hirosawa, H., Kobayashi, H., et al. 2000, PASJ, 52, 955

Hong, X., Shen, Z., An, T., \& Liu, Q. 2014, Acta Astron., 102, 217-225.

Karastergiou, A., Neri, R., \& Gurwell, M. A. 2006, ApJS, 164, 552

Kardashev, N. S., Kovalev, Y. Y., \& Kellermann, K. I. 2012, The Radio Science Bulletin No 343, 22

Keto, E. 1997, ApJ, 475, 843

Kogan, L. 1997, in ALMA memo No. 171

Liu, L., Jiang, W., Zheng, W., et al. 2019, AJ, 157, 138

Liu, Q. H., et al. 2016, Sci. China Phys. Mech. Astron. 46, 069501

Ma, C., Arias, E. F., Bianco, G., et al. 2009, IERS Technical Note, 35

Nan, R., Li, D., Jin, C., et al. 2011, International Journal of Modern Physics D, 20, 989

Park, J., Hada, K., Kino, M., et al. 2019, ApJ, 887, 147

Schartner, M. \& Böhm, J. 2019, PASP, 131, 084501

Schuh, H., \& Behrend, D. 2012, Journal of Geodynamics, 61, 68

Shepherd, M. C. 1997, Astronomical Data Analysis Software and Systems VI, 77

Storn, R and Price, K, 1997, Journal of Global Optimization, 11, 341

Su, Y., Nan, R. D., Peng, B., et al. 2004, A\&A, 414, 389

Thompson, A. R., Moran, J. M., \& Swenson, G. W., Jr. 2001, "Interferometry and synthesis in radio astronomy by A. Richard Thompson, James M. Moran, and George W. Swenson, Jr. 2nd ed. New York : Wiley, c2001.xxiii, 692 p. : ill. ; 25 cm. ”A Wiley-Interscience publication.“ Includes bibliographical references and indexes. ISBN : $0471254924 "$

Zheng, W., Huang, Y., Chen, Z., et al. 2014, International VLBI Service for Geodesy and Astrometry 2014 General Meeting Proceedings: "VGOS: The New VLBI Network", Eds. Dirk Behrend, Karen D. Baver, Kyla L. Armstrong, Science Press, Beijing, China, ISBN 978-7-03-042974-2, 2014, p. 466-472, 466 
This manuscript was prepared with the RAA LATEX macro v1.2. 